\title{
BMJ Open Treatment effect of memantine on survival in dementia with Lewy bodies and Parkinson's disease with dementia: a prospective study
}

\author{
Kajsa Stubendorff, ${ }^{1,2}$ Victoria Larsson, ${ }^{1}$ Clive Ballard, ${ }^{3}$ Lennart Minthon, ${ }^{1}$ \\ Dag Aarsland, ${ }^{4,5}$ Elisabet Londos ${ }^{1}$
}

To cite: Stubendorff $\mathrm{K}$, Larsson V, Ballard C, et al. Treatment effect of memantine on survival in dementia with Lewy bodies and Parkinson's disease with dementia: a prospective study. BMJ Open 2014;4: e005158. doi:10.1136/ bmjopen-2014-005158

- Prepublication history for this paper is available online. To view these files please visit the journal online (http://dx.doi.org/10.1136/ bmjopen-2014-005158).

Received 28 February 2014 Revised 11 June 2014 Accepted 12 June 2014

CrossMark

For numbered affiliations see end of article.

Correspondence to Dr Kajsa Stubendorff; Kajsa.Stubendorff@med.lu.se

\section{ABSTRACT}

Objective: To investigate the effect on survival of treatment with memantine in patients with dementia with Lewy bodies (DLB) and Parkinson's disease with dementia (PDD).

Methods: 75 patients with DLB and PDD were included in a prospective double-blinded randomised placebo-controlled trial (RCT) of memantine, of whom long-term follow-up was available for 42. Treatment response was recorded 24 weeks from baseline and measured by Clinical Global Impression of Change (CGIC). The participants were grouped as responders (CGIC 1-3) or non-responders (CGIC 4-7). The 24-week RCT was followed by open-label treatment and survival was recorded at 36 months.

Results: After 36-month follow-up, patients in the memantine group had a longer length of survival compared with patients in the placebo group (log rank $x^{2}=4.02, p=0.045$ ). Within the active treatment group, survival analysis 36 months from baseline showed that the memantine responders, based on CGIC, had higher rates of survival compared with the non-responders (log rank $\left.x^{2}=6.595, p=0.010\right)$. Similar results were not seen in the placebo group.

Conclusions: Early treatment with memantine and a positive clinical response to memantine predicted longer survival in patients with DLB and PDD. This suggests a possible disease-modifying effect and also has implications for health economic analysis. However, owing to the small study sample, our results should merely be considered as generating a hypothesis which needs to be evaluated in larger studies.

Trial registration number: ISRCTN89624516.

\section{INTRODUCTION}

Dementia with Lewy bodies and Parkinson's disease dementia

Dementia with Lewy bodies (DLB) and Parkinson's disease with dementia (PDD) are progressive neurodegenerative disorders, and together they account for $15-20 \%$ of all people with dementia. ${ }^{1}$ Pathologically, they

\section{Strengths and limitations of this study}

- The prospective design and the longitudinal follow-up are the strengths of this study. A follow-up time of 36 months is unique in clinical trials on dementia with Lewy bodies/Parkinson's disease with dementia (DLB/PDD).

- We use the Clinical Global Impression of Change (CGIC) to assess the treatment effect of memantine. With respect to the heterogeneous manifestations of DLB/PDD, we argue that CGIC is a robust and clinically meaningful tool to rate drug effect, and its use is therefore a strength of this study.

- The small sample size is a clear limitation of this study. Multivariate Cox regression analysis to adjust for possible covariates with effect on survival was not feasible due to the small sample size.

- Our sample consists of the Swedish population of a multicentre randomised placebo-controlled trial (RCT), and despite separate randomisation at all three centres, this subgroup analysis generates a possible selection bias.

are both $\alpha$-synucleinopathies, and clinically they have a similar profile with pronounced attentional, visuospatial and executive dysfunction, visual hallucinations, cognitive fluctuations, parkinsonism and sleep disturbances such as excessive daytime sleepiness and rapid eye movement sleep behavioural disorder. DLB and PDD are separated into two entities based on temporal differences in the emergence of symptoms. Even though many studies are aiming to further understand the differences, DLB and PDD seem to have considerable similarities and they are commonly considered as two diseases on a spectrum. ${ }^{23}$

\section{Treatment}

Currently, the only therapy licensed for the treatment of PDD is the cholinesterase 
inhibitor (ChEI) rivastigmine, based on randomised placebo-controlled trial (RCT) evidence of modest but significant benefits in cognition, function, global outcome and neuropsychiatric symptoms. ${ }^{3}$ To detect possible benefits in long-term outcome measures, a longer follow-up with open-label treatment is fundamental. Memantine is an N-methyl-D-aspartate (NMDA) receptor antagonist. It affects the glutamatergic neuronal transmission and prevents the toxic effects of raised concentration of the excitatory neurotransmitter glutamate. ${ }^{4}$ There is a known alteration of glutamatergic markers in DLB, ${ }^{5}$ and thus memantine may be beneficial in these patients, potentially by a neuroprotective and disease-modifying effect. ${ }^{6}$ Findings by Uitti et $a l^{7}$ suggest that treatment with another NMDA antagonist, amantadine, improves survival in patients with Parkinson's disease (PD) and other parkinsonian syndromes. At this time, there are four RCTs of the effect of memantine on cognition in $\mathrm{PDD}^{8-11}$ where two of them also included patients with DLB. ${ }^{8}{ }^{9}$ Memantine was well tolerated and all studies found positive effects from treatment, but consistent benefits across the studies are only evident on global outcome. Follow-up studies on the same population have also shown additional benefits on sleep disturbances ${ }^{12}$ and quality of life. ${ }^{13}$ There are some indications that memantine may have mechanisms of action which may potentially confer disease-modifying effects, ${ }^{4}$ but this has not been established in clinical studies.

\section{Survival}

Despite the relatively high prevalence and the great impact of DLB and PDD on patients, caregivers and society, there are few studies focusing on survival and on the rate and pattern of the cognitive and functional decline. Older age at onset, associated Alzheimer pathology, ${ }^{14}$ high levels of cerebrospinal total $\tau^{15}$ and severe autonomic dysfunction ${ }^{16}$ have been suggested as potential predictors of a poor prognosis in DLB/PDD. Reliable prognostic markers would be of value for patients and their families and would facilitate clinical planning, but the establishment of such markers has turned out to be problematic for several reasons. The complex nature of DLB and PDD, including a high degree of intraindividual variability in the clinical course $^{17}$ as well as day-to-day variations in clinical status, poses great demands on study design, sample size, follow-up time and statistical methods. To identify differences in survival between clinical subgroups based on treatment response may be one approach in the search for prognostic markers.

\section{Aim}

Based on a long-term follow-up of participants in a placebo-controlled RCT of memantine in DLB and PDD,${ }^{8}$ this study aims to investigate the prognostic value of early treatment with memantine and a positive response to treatment with memantine on 3-year survival in patients with DLB/PDD.

\section{MATERIALS AND METHODS \\ Study population}

This longitudinal prospective study is a continuation of a double-blinded 24-week RCT conducted in 2005-2008. ${ }^{8}$ The original study included 75 patients with mild-to-moderate DLB or PDD (Mini-Mental State Examination (MMSE) score 12 points or higher), recruited from psychiatric, memory and neurological outpatient clinics in Norway, UK and Sweden. All patients fulfilled the clinical diagnostic criteria according to the UK Parkinson's Disease Society Brain Bank and subsequently developed dementia more than a year from onset of motor symptoms (Diagnostic and Statistical Manual of Mental Disorders Fourth Edition (DSM IV); APA, 1994), or met the revised consensus criteria for DLB. ${ }^{18}$ Patients with other brain disorders, recent major changes in health status, major depression, moderate-to-severe renal impairment, heart disease, pulmonary disease, hepatic impairment or known allergy to memantine were excluded. In addition to the comprehensive clinical assessment, ${ }^{8}$ patients were examined with routine blood tests and imaging of the brain (DAT scan) to support diagnosis.

In the original study, patients were assigned to placebo or memantine treatment (20 mg daily) and assessed at baseline, 12 and 24 weeks. All three samples (from Norway, the UK and Sweden) were randomised separately, in order to allow further studies on either population. Only the Swedish centre continued the follow-up with a 4-week washout period followed by open-label treatment and ordinary yearly clinical visits within a structured follow-up programme at the clinic. Hence, the population in this study constitutes the 32 patients (16 DLB, 16 PDD) from the Swedish population (total $\mathrm{n}=42$ ) in the original study who completed the 24-week follow-up (figure 1). All 32 were enrolled in the openlabel follow-up. Seventeen (94\%) of the 18 patients in the original memantine group received memantine during the open-label follow-up. Twelve $(86 \%)$ of the 14 patients in the original placebo group received memantine during the open-label follow-up.

Randomisation was kept strictly double-blinded during the RCT (24 weeks) and during washout (4 weeks), but not during the open-label treatment. Discontinuation of the double-blinded medication was performed by the end of the RCT without sequentially decreasing the doses. The open-label medication doses were increased during a titration period of 4 weeks until reaching $20 \mathrm{mg}$ daily.

At baseline, anamnestic information, blood samples and blood pressure measurements disclosed information on concomitant diabetes, heart disease, hypertension, orthostatic hypotension and cancer. 


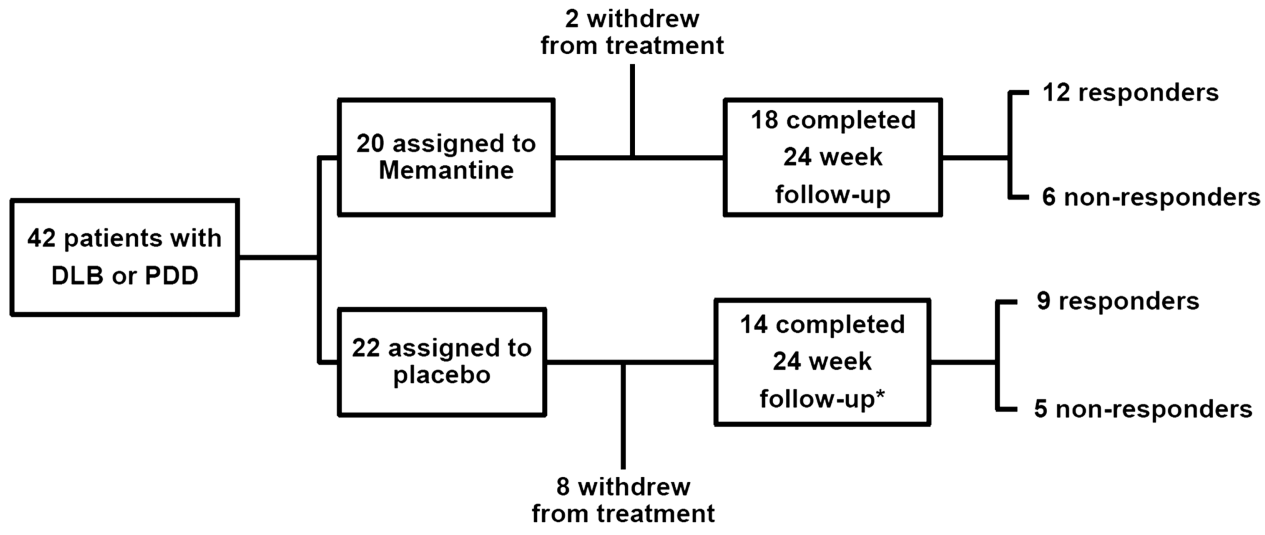

Figure 1 Trial profile of the study population (Swedish population in the original RCT). See also figure 3 for further management of the 14 patients in the placebo group. DLB, dementia with Lewy bodies; Parkinson's disease with dementia; RCT, randomised placebo-controlled trial.

\section{Treatment response and grouping}

In the original RCT, treatment response was measured at week 24 by Clinical Global Impression of Change (CGIC), which was rated based on a clinical interview with the patient and their caregiver. CGIC is a categorical scale ranging from 1 to 7 , with a low score indicating clinical improvement (figure 2). In this study, patients in the memantine and placebo groups were grouped as responders or non-responders; patients with CGIC 1-3 were responders and CGIC 4-7 were non-responders. Twelve $(67 \%)$ of the 18 patients who received memantine were responders and 6 were non-responders. In the placebo group, $9(64 \%)$ were responders and 5 were non-responders (figure 1).

Twelve of the 14 patients in the original placebo group received memantine during the open-label follow-up. After 24 weeks on treatment (54 weeks from baseline), they were again assessed with CGIC. We regrouped these 12 patients into responders $(n=3)$ and non-responders $(\mathrm{n}=9)$ based on the 54-week CGIC (figure 3).

\section{Outcome}

In this study, outcome was recorded 36 months from baseline. Survival was the only outcome measure.

\begin{tabular}{|l|l|}
\hline CGIC score & \\
\hline $\mathbf{1}$ & Substantial improvement \\
\hline $\mathbf{2}$ & Moderate improvement \\
\hline $\mathbf{3}$ & Minimum improvement \\
\hline $\mathbf{4}$ & No change \\
\hline $\mathbf{5}$ & Minimum worsening \\
\hline $\mathbf{6}$ & Moderate worsening \\
\hline $\mathbf{7}$ & Substantial worsening \\
\hline
\end{tabular}

Figure 2 Description of the Clinical Global Impression of Change (CGIC) score system.

\section{Statistics}

Statistical analyses were performed using IBM SPSS Statistics 20. Comparisons were made between participants in the memantine and placebo groups and also between responders and non-responders in the memantine and placebo groups, respectively. All binary variables were compared using $\chi^{2}$ tests. Owing to the small number of participants in this study, non-parametric statistics (Mann-Whitney $U$ test) were used to detect significant differences in groups for normally and non-normally distributed continuous variables. Kaplan-Meier curves were performed to compare survival between the two groups. Multivariate analysis to adjust for possible confounders was not feasible due to the small sample size. The $p$ values were exclusively twosided and the level of significance defined as less than 0.05 .

\section{Ethical statement}

The RCT was conducted in accordance with the Declaration of Helsinki as revised in 2000 , as was the open-label extension study. Renewed written informed consent was obtained from patients and their caregivers before entering the open-label extension.

\section{RESULTS}

Comparisons were made at baseline between patients in the memantine group and the placebo group, as well as between responders and non-responders in the memantine and placebo groups, respectively (table 1 ). Age at baseline, gender or disease duration did not differ between groups. For patients with PDD, the difference in duration of PD before dementia was non-significant (data not shown). Concomitant medications (antiparkinsonian medication, ChEIs, neuroleptics, anxiolytics, hypnotics and antidepressants) were used without significant differences, but there was a clinically meaningful difference in the proportion receiving ChEI treatment $(33 \%$ in the memantine vs $71 \%$ in the placebo 


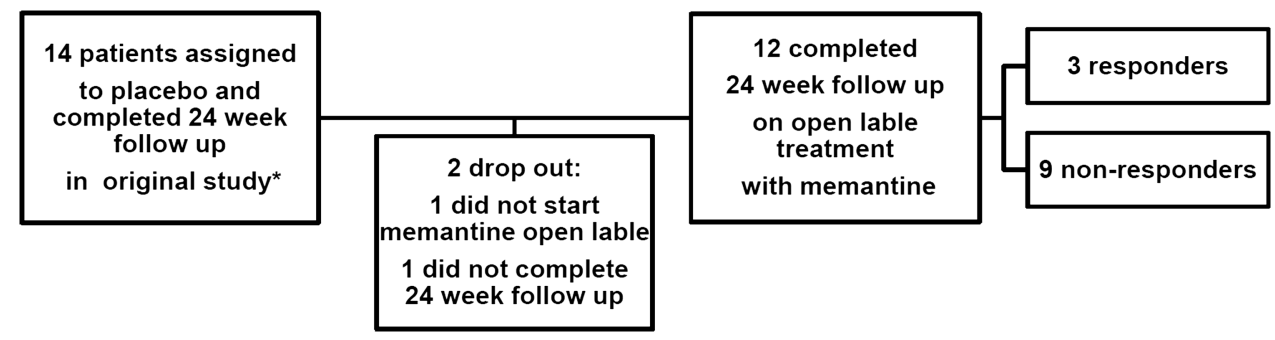

Figure 3 Trial profile for further management of the original placebo group during the open-label treatment follow-up. See also figure 1.

group). The dose of levodopa was equal. MMSE scores at baseline were equal. There were no differences in the degree of parkinsonism measured by the Unified Parkinson's Disease Rating Scale (UPDRS), or frequency of orthostatic hypotension. Presence of diabetes, heart disease, hypertension and cancer was investigated, but there were no differences in the burden of comorbidity not related to dementia (data not shown).

\section{Survival}

Fifteen $(47 \%)$ of the 32 participants died during the follow-up. In the memantine group, 5 (28\%) of the 18 patients died, and in the placebo group 10 of the 14 $(71 \%)$ patients died $\left(x^{2}=6.03, p=0.03\right)$. Patients in the original memantine group had a better 3-year survival compared with the placebo group $\left(\log r a n k x^{2}=4.021\right.$, $\mathrm{p}=0.045$; figure 4 ).

In the memantine group, $1(8 \%)$ of the 12 responders and $4(67 \%)$ of the 6 non-responders died during the follow-up $\left(\mathrm{x}^{2}=6.785, \mathrm{p}=0.02\right)$. The Kaplan-Meier curves in figure 5 show the influence of a positive treatment response on survival. Patients with a positive treatment response to memantine, recorded 24 weeks after baseline, had a significantly longer survival compared with non-responders $\left(\log\right.$ rank $\mathrm{x}^{2}=6.595, \mathrm{p}=0.010$; figure $\left.5 \mathrm{~A}\right)$. There was no significant difference in survival between responders and non-responders in the placebo group (figure 5B).

With the intention of strengthening our findings, we compared 3-year survival between responders and nonresponders in the original placebo group, based on the 54-week CGIC, when they all had been on open-label treatment with memantine for 24 weeks. One $(33 \%)$ of the 3 responders and 7 (78\%) of the 9 non-responders died during the 3-year follow-up, but no difference was found in the survival analysis (figure 6).

\section{DISCUSSION}

The results of this study show that patients with DLB/ PDD treated with memantine had a better 3-year survival, compared with patients treated with placebo, despite both groups receiving subsequent open-label treatment with memantine after 6 months. Within the memantine group, patients who responded positively to treatment had a better survival compared with non- responders. In the placebo group, the frequency of responders was notably high, but was not associated with improved survival. This builds on previous RCT evidence indicating a global benefit from memantine treatment in patients with DLB/PDD.

According to survival analysis, patients in the placebo group died to a greater extent during the 3-year follow-up compared with those originally assigned to the memantine group. However, $29(90 \%)$ of the 32 patients were on treatment with memantine during open-label treatment, and therefore memantine alone cannot be said to enhance survival. Perhaps this could indicate that patients can benefit from treatment with memantine, but only when it is introduced early in the clinical course. Furthermore, this is in line with the study on the same population by Johansson et al, ${ }^{19}$ who noticed a global improvement in the placebo group as well as in the memantine group during the 30 weeks of open-label follow-up, but the change in CGIC was not significant.

In the small sample of our study, we could not find any differences in baseline characteristics between responders and non-responders in the memantine group (table 1). However, a critical factor characterising a responder subgroup may not be clinically detectable, but could possibly be established by cerebrospinal fluid analysis or by neuroimaging methods.

The underlying mechanisms to why a positive response to memantine implicated longer survival can only be hypothesised at this point. Possible explanations include that the symptomatic benefit translated into better physical health and lower mortality (ie, reduced infections, thrombosis, falls, etc), or that the responder status is a marker of an overall better prognosis, although the lack of difference in the placebo group argues against these explanations. It cannot be ruled out that memantine in some way has direct positive effects on survival. Possibly, if patients or subgroups of patients with DLB/PDD receive memantine at an early stage in their disease, the treatment may target and release cognitive and physical reserve capacity, leading to increasing survival time.

We have no reason to believe that memantine can prevent any of the main causes of death in dementiacachexia/dehydration, pneumonia (from associated somatic decline or swallowing problems) and cardiovascular disorders. ${ }^{20}{ }^{21}$ In our study sample, the mean age 
Table 1 Demographics

\begin{tabular}{|c|c|c|c|c|c|c|c|c|c|}
\hline & \multicolumn{3}{|c|}{ Total study population $(n=32)$} & \multicolumn{3}{|c|}{ Memantine group $(n=18)$} & \multicolumn{3}{|c|}{ Placebo group $(n=14)$} \\
\hline & $\begin{array}{l}\text { Memantine } \\
\text { group }(n=18)\end{array}$ & $\begin{array}{l}\text { Placebo } \\
\text { group } \\
(n=14)\end{array}$ & $p$ Value & $\begin{array}{l}\text { Responders } \\
(\mathrm{n}=12)\end{array}$ & $\begin{array}{l}\text { Non-responders } \\
(\mathrm{n}=6)\end{array}$ & p Value & $\begin{array}{l}\text { Responders } \\
(\mathrm{n}=9)\end{array}$ & $\begin{array}{l}\text { Non-responders } \\
(\mathrm{n}=5)\end{array}$ & p Value \\
\hline Age at baseline (years) & $74.3 \pm 5.4$ & $76.2 \pm 4.5$ & ns & $73.8 \pm 5.5$ & $75.2 \pm 5.6$ & ns & $76.5 \pm 4.7$ & $75.6 \pm 4.6$ & ns \\
\hline Gender (M:F) & $15: 3$ & $9: 5$ & ns & $9: 3$ & $6: 0$ & ns & $6: 3$ & $3: 2$ & ns \\
\hline Diagnosis (DLB:PDD) & $8: 10$ & $8: 6$ & ns & $5: 7$ & $3: 3$ & ns & $5: 4$ & $3: 2$ & ns \\
\hline Disease duration (years) & $5.8 \pm 3.5$ & $6.2 \pm 3.5$ & ns & $6.3 \pm 3.4$ & $4.8 \pm 3.3$ & ns & $6.3 \pm 3.3$ & $6.0 \pm 4.2$ & ns \\
\hline \multicolumn{10}{|c|}{ Concomitant medication, number of yes (\%) } \\
\hline Antiparkinsonian treatment & $14(78 \%)$ & $12(86 \%)$ & ns & $10(83 \%)$ & $4(67 \%)$ & ns & $8(89 \%)$ & $4(80 \%)$ & ns \\
\hline Levodopa dose (mg/day) & $625 \pm 399$ & $468 \pm 308$ & ns & $528 \pm 154$ & $883 \pm 759$ & ns & $407 \pm 333$ & $575 \pm 266$ & ns \\
\hline Cholinesterase inhibitors & $6(33 \%)$ & $10(71 \%)$ & ns & $3(25 \%)$ & $3(50 \%)$ & ns & $8(89 \%)$ & $2(40 \%)$ & ns \\
\hline Neuroleptics & $3(17 \%)$ & $4(29 \%)$ & $\mathrm{ns}$ & $1(8 \%)$ & $2(33 \%)$ & ns & $3(33 \%)$ & $1(20 \%)$ & ns \\
\hline Anxiolytics & $4(22 \%)$ & $2(14 \%)$ & $\mathrm{ns}$ & $2(17 \%)$ & $2(33 \%)$ & ns & $0(0 \%)$ & $2(40 \%)$ & ns \\
\hline Hypnotics & $7(39 \%)$ & $3(21 \%)$ & ns & $4(33 \%)$ & $3(50 \%)$ & ns & $1(11 \%)$ & $2(40 \%)$ & ns \\
\hline Antidepressants & $9(50 \%)$ & $5(36 \%)$ & ns & $6(50 \%)$ & $3(50 \%)$ & ns & $2(22 \%)$ & $3(60 \%)$ & ns \\
\hline \multicolumn{10}{|l|}{ Clinical features at baseline } \\
\hline MMSE at baseline & $20.7 \pm 4.0$ & $19.7 \pm 4.7$ & $\mathrm{~ns}$ & $20.4 \pm 3.8$ & $21.3 \pm 4.7$ & ns & $19.4 \pm 4.7$ & $21.2 \pm 4.2$ & ns \\
\hline UPDRS at baseline & $34 \pm 13$ & $41 \pm 12$ & $\mathrm{~ns}$ & $34 \pm 14$ & $34 \pm 10$ & ns & $38 \pm 8$ & $45 \pm 17$ & ns \\
\hline $\begin{array}{l}\text { Orthostatic hypotension, } \\
\text { number of yes }(\%)\end{array}$ & $11(61)$ & $7(50)$ & ns & $6(50)$ & $5(83)$ & ns & $5(63)$ & $2(40)$ & ns \\
\hline
\end{tabular}




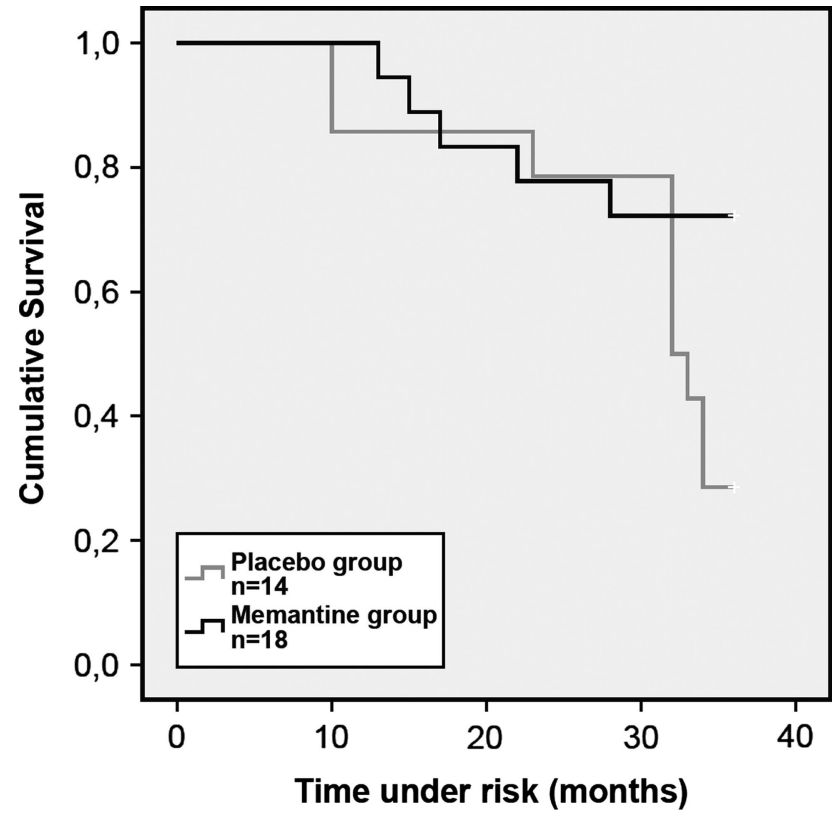

Figure 4 Kaplan-Meier estimates of the rate of survival in patients with dementia with Lewy bodies and Parkinson's disease with dementia following 24-week placebo-controlled double-blinded treatment with memantine. The group of patients that received treatment with memantine during the first 24 weeks had a higher 3-year survival rate compared with patients in the placebo group (log rank $\left.x^{2}=4.021, p=0.045\right)$.

in responders versus non-responders was equal, and there were no differences in drug use or burden of comorbidity. Even though there was no statistical difference, a lower percentage of the patients in the responder group received ChEI. This is related to the relatively greater number of patients with PDD in this group, as patients with PDD are often recruited from neurological outpatient clinics where the use of ChEI is traditionally lower compared with memory clinics.

A potential limitation that our study shares with all long-term follow-up studies on patients with neurodegenerative diseases is the well-known bias due to preselected clinical populations. For patients with Alzheimer disease, it is shown that those who participate in longterm clinical trials with open-label extensions tend to be younger, have a higher level of education and a better financial situation than patients not enrolled in trials. $^{22}{ }^{23}$ Our sample consists of the Swedish population out of a multicentre RCT, and despite separate randomisation at all three centres, this subgroup analysis generates another possible selection bias.

The small sample size of our study is a clear limitation. There is a risk that misclassification of individual patients would have a major impact on the result. Cox regression analyses to find possible covariates with effect on survival are not possible due to the small sample size.
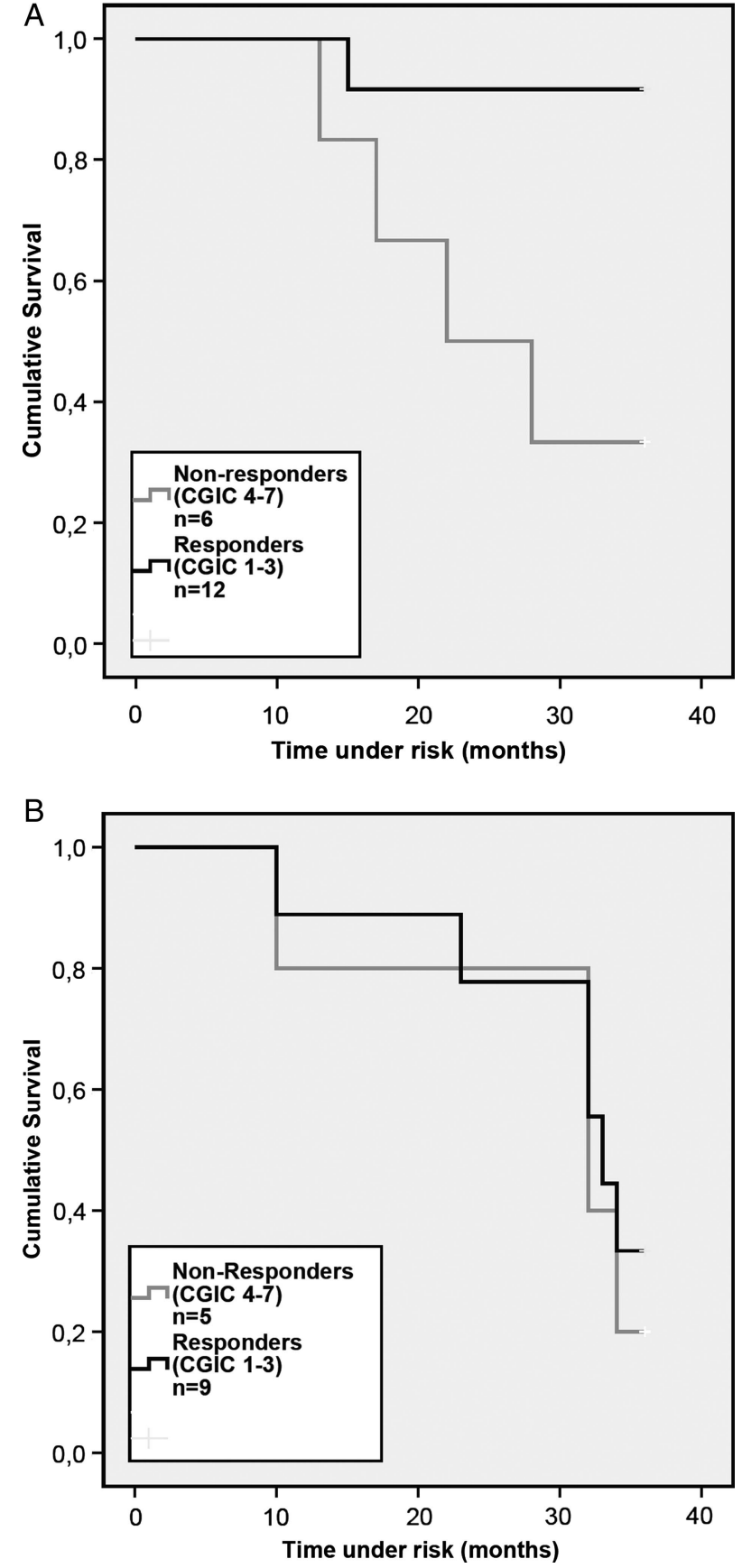

Figure 5 (A) Kaplan-Meier estimates of the rate of survival in patients with DLB and PDD following 24-week double-blinded treatment with memantine. The group of patients that responded positively to treatment after 24 weeks had a higher 3-year survival rate compared with non-responders (log rank $\left.x^{2}=6.595, p=0.010\right)$. (B) Kaplan-Meier estimates of the rate of survival in patients with DLB and PDD following double-blinded treatment with placebo. There was no difference in survival between the patients who responded positively to placebo compared with the non-responders (log rank $\left.x^{2}=0.161, p=0.689\right)$. CGIC, Clinical Global Impression of Change; DLB, dementia with Lewy bodies; Parkinson's disease with dementia. 


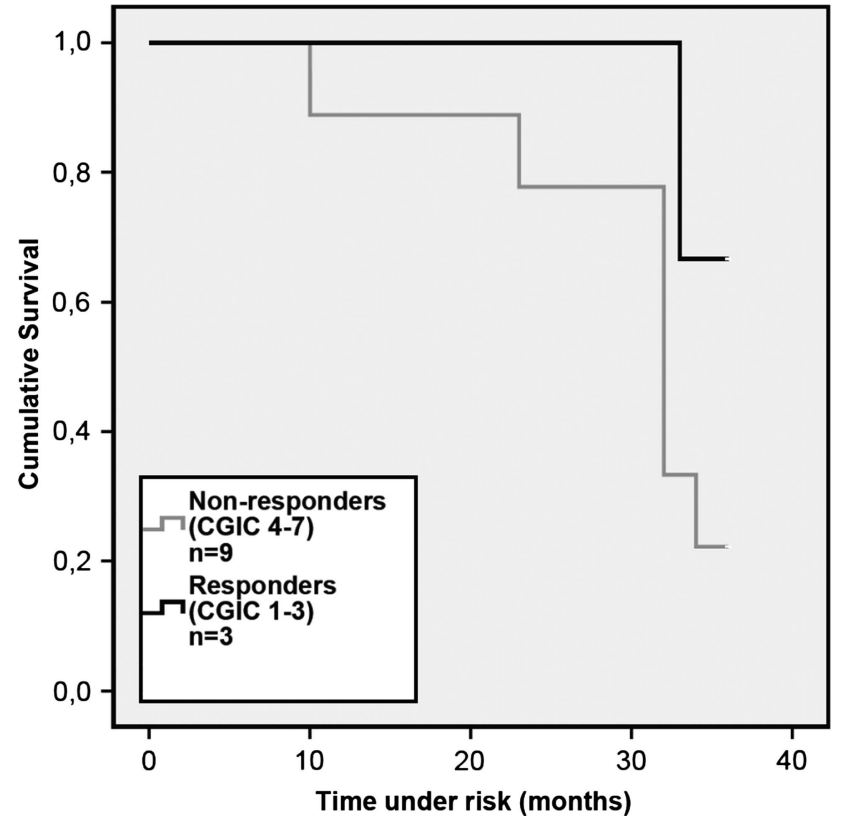

Figure 6 Kaplan-Meier estimates of the rate of survival in patients with DLB and PDD following 6-month open-label treatment with memantine. There was no difference in survival between the patients who responded positively to treatment compared with the non-responders (log rank $x^{2}=1.834$, $\mathrm{p}=0.176)$. CGIC, Clinical Global Impression of Change; DLB, dementia with Lewy bodies; Parkinson's disease with dementia.

Nevertheless, significant findings in a small-size sample cannot be disregarded, and long-term follow-up studies are demanding to perform due to the fragility of these patients. A higher rate of dropout during follow-up in the placebo group compared with the memantine group generated an imbalance between the groups. However, this difference could be due to a lack of treatment effect among patients in the placebo group, and would then be in line with our findings.

In our study, $15(47 \%)$ of the 32 patients died during the 3-year follow-up, revealing the terminal course of DLB and PDD. In Sweden, a postmortem examination is no longer included as standard procedure in the management of patients with DLB/PDD, and we had no intention to confirm the clinical diagnosis of all patients in this study. However, three of the patients included in this study have been examined postmortem, and the clinical diagnosis was confirmed in all three cases.

We used CGIC as the measurement tool when grouping the patients into responders or non-responders. A global scale, like CGIC, is recommended by European Medicines Agency (EMEA) to be used in all clinical trials on dementia. ${ }^{24}$ It allows a subjective integrative judgement by the clinician on the patient's symptoms and performance, as opposed to assessing various functions by a set of tests. With respect to the heterogeneous manifestations of DLB/PDD, consisting of cognitive, functional, motor and psychiatric deficits, we argue that
CGIC is a robust and clinically meaningful tool to rate the drug effect and its use is therefore a strength of this study.

Our only outcome measure is survival, which, compared with the change or rate of deterioration in scores on cognitive, functional or behavioural scales, is a solid end point. The natural course of DLB/PDD includes temporary changes over time, and changes in clinical scores can be difficult to interpret. To have survival as the only outcome measure does not exclude patients who are not able to perform the testing, and therefore the number of dropouts during follow-up is minimised.

In this setting, we cannot propose a disease-modifying effect of memantine in patients with DLB/PDD. Our findings are based on clinical data alone, which is insufficient to prove a true disease-modifying effect. To prove such effect, biomarkers in direct association with the underlying disease process are obligate, and our study highlights the need for a validated biomarker programme to detect subgroups of patients and to demonstrate a disease-modifying drug effect, that is, changes in the rate of neurodegeneration. Our intention is to present a new idea, not to report conclusive data. This study is the first to investigate the prognostic value of a positive response to treatment in dementia. Prospective longitudinal clinical trials on patients with $\mathrm{DLB} / \mathrm{PDD}$ are rare and therefore valuable. A follow-up time of 36 months is unique in clinical trials on these patients.

In conclusion, our interpretation of our results indicates that memantine might enhance survival, and within this group, patients who respond to treatment positively might survive longer. This has an effect on patients, clinicians and health economic analysis. We hope that our findings can be an inspiration for future trials of a larger scale.

\section{Author affiliations}

${ }^{1}$ Clinical Memory Research Unit, Department of Clinical Sciences, Lund University, Malmö, Sweden

${ }^{2}$ Department of Rheumatology, Skaraborg Central Hospital, Skövde, Sweden ${ }^{3}$ Wolfson Centre for Age Related Diseases, King's College London,

London, UK

${ }^{4}$ Center for Age-Related Medicine, Stavanger University Hospital, Stavanger, Norway

${ }^{5}$ Department of NVS, Neurobiology Ward Sciences and Society, Alzheimer's Disease Research Center, Karolinska Institutet, Stockholm, Sweden

Acknowledgements The authors thank the patients and their relatives for their cooperation in this study. They also thank Eva Falk-Langebro for the management of the patients. CB would like to thank the Biomedical Research Unit for dementia at King's College London for supporting his involvement in the study.

Contributors EL conceived, designed and performed the experiments. KS, VL and EL analysed the data. All authors contributed to the interpretation of data, drafted or revised the paper.

Funding Swedish Research Council (nr 523-2010-520) and the Swedish Parkinson Foundation gave financial support.

Competing interests DA has received honoraria and research support from H Lundbeck A/S, Novartis, GE Healthcare and Merck-Serono. CB has received 
honoraria from Acadia, Bristol-Meyers Sqidd, Eisai, Janssen, H Lundbeck A/S, Novartis and Shire. EL has received honoraria from $H$ Lundbeck A/S and Novartis for lectures.

Ethics approval The regional ethics committee at Lund University.

Provenance and peer review Not commissioned; externally peer reviewed.

Data sharing statement No additional data are available.

Open Access This is an Open Access article distributed in accordance with the Creative Commons Attribution Non Commercial (CC BY-NC 3.0) license, which permits others to distribute, remix, adapt, build upon this work noncommercially, and license their derivative works on different terms, provided the original work is properly cited and the use is non-commercial. See: http:// creativecommons.org/licenses/by-nc/3.0/

\section{REFERENCES}

1. Lippa CF, Duda JE, Grossman M, et al. DLB and PDD boundary issues: diagnosis, treatment, molecular pathology, and biomarkers. Neurology 2007;68:812-19.

2. Aarsland D, Ballard CG, Halliday G. Are Parkinson's disease with dementia and dementia with Lewy bodies the same entity? J Geriatr Psychiatry Neurol 2004;17:137-45.

3. Ballard C, Kahn Z, Corbett A. Treatment of dementia with Lewy bodies and Parkinson's disease dementia. Drugs Aging 2011;28:769-77.

4. Thomas SJ, Grossberg GT. Memantine: a review of studies into its safety and efficacy in treating Alzheimer's disease and other dementias. Clin Interv Aging 2009;4:367-77.

5. Dalfo E, Albasanz JL, Martin M, et al. Abnormal metabotropic glutamate receptor expression and signaling in the cerebral cortex in diffuse Lewy body disease is associated with irregular alpha-synuclein/phospholipase C (PLCbeta1) interactions. Brain Pathol 2004;14:388-98.

6. Lipton SA. The molecular basis of memantine action in Alzheimer's disease and other neurologic disorders: low-affinity, uncompetitive antagonism. Curr Alzheimer Res 2005;2:155-65.

7. Uitti RJ, Rajput AH, Ahlskog JE, et al. Amantadine treatment is an independent predictor of improved survival in Parkinson's disease. Neurology 1996;46:1551-6.

8. Aarsland D, Ballard C, Walker Z, et al. Memantine in patients with Parkinson's disease dementia or dementia with Lewy bodies: a double-blind, placebo-controlled, multicentre trial. Lancet Neurol 2009;8:613-18.

9. Emre M, Tsolaki M, Bonuccelli U, et al. Memantine for patients with Parkinson's disease dementia or dementia with Lewy bodies: a randomised, double-blind, placebo-controlled trial. Lancet Neurol 2010;9:969-77.

10. Leroi I, Overshott R, Byrne EJ, et al. Randomized controlled trial of memantine in dementia associated with Parkinson's disease. Mov Disord 2009;24:1217-21.

11. Ondo WG, Shinawi L, Davidson A et al. Memantine for non-motor features of Parkinson's disease: a double-blind placebo controlled exploratory pilot trial. Parkinsonism Relat Disord 2011;17: 156-9.

12. Larsson V, Aarsland D, Ballard C, et al. The effect of memantine on sleep behaviour in dementia with Lewy bodies and Parkinson's disease dementia. Int J Geriatr Psychiatry 2010;25: 1030-8.

13. Larsson V, Engedal K, Aarsland D, et al. Quality of life and the effect of memantine in dementia with lewy bodies and Parkinson's disease dementia. Dement Geriatr Cogn Disord 2011;32:227-34.

14. Jellinger KA, Wenning GK, Seppi K. Predictors of survival in dementia with lewy bodies and Parkinson dementia. Neurodegener Dis 2007;4:428-30.

15. Bostrom $\mathrm{F}$, Hansson $\mathrm{O}$, Blennow $\mathrm{K}$, et al. Cerebrospinal fluid total tau is associated with shorter survival in dementia with Lewy bodies. Dement Geriatr Cogn Disord 2009;28:314-19.

16. Stubendorff $\mathrm{K}$, Aarsland D, Minthon L, et al. The impact of autonomic dysfunction on survival in patients with dementia with Lewy bodies and Parkinson's disease with dementia. PLOS ONE 2012;7:e45451.

17. Gaig C, Valldeoriola F, Gelpi E, et al. Rapidly progressive diffuse Lewy body disease. Mov Disord 2011;26:1316-23.

18. McKeith IG, Dickson DW, Lowe J, et al. Diagnosis and management of dementia with Lewy bodies: third report of the DLB Consortium. Neurology 2005;65:1863-72.

19. Johansson C, Ballard C, Hansson O, et al. Efficacy of memantine in PDD and DLB: an extension study including washout and open-labe treatment. Int J Geriatr Psychiatry 2011;26:206-13.

20. Boersma F, Van Den Brink W, Deeg D, et al. Survival in a population-based cohort of dementia patients: predictors and causes of mortality. Int J Geriatr Psychiatry 1999;14:748-53.

21. Koopmans RT, van der Sterren KJ, van der Steen JT. The 'natural' endpoint of dementia: death from cachexia or dehydration following palliative care? Int J Geriatr Psychiatry 2007;22:350-5.

22. Albert SM, Sano M, Marder K, et al. Participation in clinical trials and long-term outcomes in Alzheimer's disease. Neurology 1997;49:38-43.

23. Schneider LS, Olin JT, Lyness SA, et al. Eligibility of Alzheimer's disease clinic patients for clinical trials. J Am Geriatr Soc 1997;45:923-8.

24. European Medicines Agency CfMPfHUC. Guideline on medicinal products for the treatment of Alzheimer's disease and other dementias. London, 2008. 